\title{
A NON-STATIONARY GENERALIZATION OF THE KERR CONGRUENCE
}

\author{
Vladimir V. Kassandrov ${ }^{1}$ \\ Institute of Gravitation and Cosmology, Peoples' Friendship University of Russia, \\ Miklukho-Maklaya St. 6, Moscow, 117198 Russia
}

\begin{abstract}
Making use of the Kerr theorem for shear-free null congruences and of Newman's representation for a virtual charge "moving" in complex space-time, we obtain an axisymmetric time-dependent generalization of the Kerr congruence, with a singular ring uniformly contracting to a point and expanding then to infinity. Electromagnetic and complex eikonal field distributions are naturally associated with the obtained congruence, with electric charge being necesssarily unit ("elementary"). We conjecture that the corresponding solution to the Einstein-Maxwell equations could describe the process of continious transition of the naked ringlike singularitiy into a rotating black hole and vice versa, under a particular current radius of the singular ring.
\end{abstract}

\section{Shear-free null congruences and associated symmetries and fields}

A broad class of physically relevant solutions to the Einstein or Einstein-Maxwell equations is generated by a shear-free congruence of null rays. In particular, the Schwarzchild (Reissner-Nordström) and Kerr (Kerr-Newman) solutions belong to this class. Since the times of the paper by Debney, Kerr and Schild [1], it is known that the Einstein equations are partly satisfied after substitution of the Riemannian metric

$$
g_{\mu \nu}=\eta_{\mu \nu}+h k_{\mu} k_{\nu}
$$

with a null 4-vector field $k: k_{\mu} k^{\mu}=0$ obeying the defining equations of shear-free null congruences (SFNC) in Minkowski background space with the metric $\eta_{\mu \nu}$ (as a consequence, these are automatically geodetic, composed of rectilinear rays). In the above-mentioned cases it is, moreover, possible to choose the scalar "gravitational potential" $h$ in such a way that the rest of equations are fulfilled as well. Note that properties of a congruence to be null, geodetic and shear-free are preserved by themselves under the Kerr-Schild deformation (11) of the Minkowski metric.

On the other hand, the SFNC equations may be represented in a spinorial form [2]

$$
\xi^{A} \xi^{B} \nabla_{B^{\prime} B} \xi_{A}=0
$$

for a principal 2-spinor field of the congruence $\xi=$ $\left\{\xi_{A}\right\}, A=1,2$ up to a phase factor defined by the null 4 -vector field $k_{\mu}=\xi_{A} \xi_{A^{\prime}}$. It has been in fact shown in the very work [1] that, in the Minkowski background, SFNCs carry a twistorial structure corresponding to the principal spinor $\xi$ and, as a result, they may be completely described in a purely algebraic way. Such a remarkable statement is known as the Kerr theorem 2 and, for our further purposes, can be formulated in an invariant form [3, 4].

\footnotetext{
${ }^{1}$ e-mail: vkassan@sci.pfu.edu.ru
}

Specifically, consider an arbitrary and (almost everywhere) analytical 2-surface in the 4-dimensional complex (twistor) space $\mathbf{W}=\{\xi, \tau\}$. The surface is represented implicitly through a set of two algebraic constraints

$$
\Pi^{C}(\xi, \tau)=0, \quad C=1,2,
$$

so that only two of the four twistor components are functionally independent.

Let us now make use of the twistor-defining incidence relation [2]

$$
\tau=X \xi, \quad\left(\tau^{A^{\prime}}=X^{A^{\prime} A_{1}} \xi_{A}\right),
$$

linking the two spinor constituents $\{\xi, \tau\}$ with the points $X$ of the Minkowski space $\mathbf{M}$ represented by a $2 \times 2$ Hermitean matrix $X=X^{+}$. After substitution of (4) into (3) the latter turns to be a pair of algebraic equations

$$
\Pi^{C}(\xi, X \xi)=0, \quad C=1,2,
$$

for two unknowns $\xi$, with the coordinates $\left\{X^{A^{\prime} A}\right\}$ playing the role of parameters. Successively resolving (5) at different points $X \in \mathbf{M}$, one obtains a multivalued solution $\xi(X)$ whose every branch defines, in fact, a SFNC (exactly, a sub-congruence of this type).

Moreover, the spinor found obeys an overdetermined set of four equations

$$
\xi^{B} \nabla_{B^{\prime} B} \xi_{A}=0,
$$

from which the standard form of the SFNC equations (3) results through contraction with $\xi^{A}$.

To prove this, one differentiates (5) in each of the coordinates $X^{B^{\prime} B}$ to get

$$
Q^{C A} \nabla_{B^{\prime} B} \xi_{A}=-\frac{\partial \Pi^{C}}{\partial \tau^{B^{\prime}}} \xi_{B},
$$

with the $2 \times 2$ matrix $Q^{C A}$ of the following form:

$$
Q^{C A}=\frac{d \Pi^{C}}{d \xi_{A}}=\frac{\partial \Pi^{C}}{\partial \xi_{A}}+\frac{\partial \Pi^{C}}{\partial \tau^{A^{\prime}}} X^{A^{\prime} A} .
$$


At the points defined by the condition

$$
\operatorname{det}\left\|Q^{C A}\right\|=0,
$$

the derivatives of the principal spinor are indefinite, so that (9) turns out to be the condition of a caustic locus for rays of the SFNC. On the other hand, at all regular points contracting (7) with $\xi_{B}$ one gets the aforepresented set of equations (6).

One can furthermore notice that, with respect to the ratio of components of the principal spinor $\xi$, Eqs. (2) and (6) are entirely equivalent, the latter only specifying, in addition, each of the two spinor components. This is in correspondence with the symmetries of these equations.

Indeed, the standard SFNC equations (2) as well as the incidence relation (44) preserve their form under arbitrary rescaling $\xi \mapsto \alpha(X) \xi$, and one can easily show that the gauge parameter $\alpha$ may be chosen in such a way that the transformed spinor satisfies the stronger equations (6). The latter possess a restricted reparametrization invariance [3, 4, with the parameter $\alpha$ depending on the coordinates only implicitly, via the components of the transforming spinor or its twistor counterparts, $\alpha=\alpha(\xi, \tau)$. Evidently, this restricted symmetry is also a symmetry of the algebraic constraint (5) and, geometrically, it has the meaning of an arbitrary diffeomorphism in twistor space $\{\xi, \tau\}$. For the associated electromagnetic field (see below) it manifests itself as a "weak" version of the familiar gauge symmetry.

Any SFNC allows for definition of a (complexified) electromagnetic-like field [5, 6]. Specifically, making use of spinor algebra, one can write down the SFNC equations (2) in the equivalent form [7, 8 ,

$$
\nabla_{B^{\prime}(B} \xi_{A)}=\Phi_{B^{\prime}(B} \xi_{A)}
$$

where some complex 4-vector $\Phi_{B^{\prime} B}$ comes into play, and the parentheses denote symmetrization in spinor indices. Analogously, Eqs. (6) read

$$
\nabla_{B^{\prime} B} \xi_{A}=\Phi_{B^{\prime} A} \xi_{B}
$$

(with the skew-symmetric part being defined in addition to (10)). Under rescalings of the principal spinor, the 4vector $\Phi_{B^{\prime} B}$ transforms gradient-wise and can thus be interpreted as the potential of an Abelian complex gauge field. As a consequence of the integrability conditions for (10), the field strengths

$$
\varphi_{(A B)}=\nabla_{B^{\prime}(B} \Phi_{A)}^{B^{\prime}}
$$

are anti-self-dual [6, 9]

$$
\nabla_{\left(A^{\prime} B\right.} \Phi_{\left.B^{\prime}\right)}^{B}=0
$$

and thus satisfy the homogeneous Maxwell equations

$$
\nabla_{B^{\prime}}^{A} \varphi_{(A B)}=0 \text {. }
$$

The field strengths are rescaling (gauge-) invariant and can be expressed through second-order derivatives of the only complex function $g(X)$, the ratio of two complex components, say, $g=\xi_{2^{\prime}} / \xi_{1^{\prime}}$ [9]. With respect to the projective spinor component $g(X)$, the generating constraints (5) reduce to the only one

$$
\Pi\left(g, \tau^{1}, \tau^{2}\right)=0,
$$

containing three projective twistor components. Introducing a canonical representation of coordinates on $\mathbf{M}$,

$$
X=X^{+}=\left(\begin{array}{cc}
u & w \\
p & v
\end{array}\right)=\left(\begin{array}{cc}
c t+z & x-\imath y \\
x+\imath y & c t-z
\end{array}\right),
$$

with $u, v$ being real and $p, w$ complex-conjugated, the incidence relation (4) takes the form of two equations:

$$
\tau^{1}=u+w g, \quad \tau^{2}=p+v g,
$$

so that the constraint (15) becomes an equation for the sole unknown $g$,

$$
\Pi(g, u+w g, p+v g)=0,
$$

the condition (9) for SFNC singularities (caustics) simplifies to

$$
P:=\frac{d \Pi}{d g} \equiv \frac{\partial \Pi}{\partial g}+w \Pi_{1}+v \Pi_{2}=0,
$$

and the field strengths of the associated Maxwell field (12) can be expressed explicitly through the (first and second order) derivatives $\Pi_{A}, \Pi_{A B}$ of generating function $\Pi$ with respect to its twistor arguments $\tau^{A}[3,4$ :

$$
\varphi_{(A B)}=\frac{1}{P}\left(\Pi_{A B}-\frac{d}{d g}\left\{\frac{\Pi_{A} \Pi_{B}}{P}\right\}\right) .
$$

Note that, in the gauge used, from the sole constraint (18) one obtains a projective spinor component $g(X)$ that defines a SFNC. It is, in fact, the content of the Kerr theorem that any SFNC on $\mathbf{M}$ may be obtained in such an algebraic way. In a gauge-invariant form, the same is true if one starts from the pair of algebraic constraints (3).

Remarkably, the ratio $g(X)$ of spinor components for any SFNC (which may be obtained from (18) with some generating function $\Pi$ ) identically satisfies the two fundamental Lorentz-invariant equations, both together, namely, the linear wave equation $\nabla^{2} g=0$ and the nonlinear eikonal equation $(\nabla g)^{2}=0$. In a spinorial gauge-invariant form, the corresponding relations have been written down in 8 .

As to the introduced Maxwell field, it is exceptional in a number of properties. Contrary to some other types of Maxwell fields related to SFNC and introduced some time ago, say, in [10, 2, 7, 13, the above introduced field is gauge-invariant, generally non-null and does not require explicit integration of Maxwell's equations themselves. Though this field almost everywhere obeys the homogeneous ("free") Maxwell equations, it nonetheless defines some effective singular "sources" located at the caustic points (19) of the congruence. Remarkably, all 
these solutions to Maxwell equations are generated by arbitrary twistor functions $\Pi(\mathbf{W})$ and can be obtained in a purely algebraic way, via resolving (18) and successive calculation of the strengths (20).

On the other hand, only a distinguished subclass of solutions to the Maxwell's equations may be obtained in such a way, and for this reason a sort of "selection rules" naturally arise. In particular, for any SFNC any (bound in 3-space) singularity of the electric field (20), defined by the condition (19), is either neutral or carries an electric charge multiple to a minimal "elementary" charge possible in the model presented. The "elementary" charge arises as that of a ringlike singularity of the fundamental Kerr-like solution considered below. The general theorem of charge quantization proved in 15 makes use of considerations resembling those in Dirac's theory of magnetic monopoles.

It is noteworthy that, in addition to the eikonal, Maxwell and metric (curvature) fields, it is possible to associate with any SFNC (i.e., with any generating twistor function) a pair of 2-spinors, each satisfying the massless Weyl equations [15] and the ("restricted" $S L(2, \mathbb{C})$ ) Yang-Mills type gauge field equations [6, 9] (see a discussion in [3]). We thus see that a shearfree null congruence (exactly, a closed system of subcongruences generating by a unique complex structure) in a Minkowski or Kerr-Schild background is really a fundamental geometrophysical object giving itself rise to a whole system of massless relativistic fields and singular "particlelike" formations, the latter revealing some properties of real quantum particles. In the algebrodynamical paradigm, as it has been demonstrated in [5, 6, 4, the naturally formulating conditions of biquaternionic analyticity ("generalized Cauchy-Riemann equations") are in fact equivalent to the defining equations of SFNC.

\section{Newman's "virtual charge" and the complex null cone representation}

Shear-free congruences of null rays are especially important because it is these congruences that are generated by an arbitrary moving charge through its local light cone. Making use of such a congruence, for simplest cases one can integrate the Einstein or Einstein-Maxwell set of equations and thus associate the electromagnetic and metric (curvature) field with a moving pointlike charge/mass [11, 12. Newman et al. [13, 14] generalized this procedure by consideration of the complex extension $\mathbb{C M}$ of the Minkowski space M. A "virtual" pointlike singularity "moving" therein along a "trajectory"

$$
z_{\mu}^{0}(\sigma), \quad \sigma \in \mathbb{C}, \quad \mu=0,1,2,3
$$

generates the complexified Lienard-Wiechert electomagnetic and gravitational fields obtained by integration of the correspondent field equations and subsequent restriction of the solutions onto the real space-time "cut" M.

On the other hand, one can suggest an alternative, purely algebraic and direct method of generating particular solutions for, say, Maxwell and eikonal fields described in the previous section: these correspond to Newman's "virtual" pointlike singularity in complex space. Specifically, consider the case of the generating function (15) such that it is identically nullified by the 1-parametric substitution

$$
z_{\mu}=z_{\mu}^{0}(\sigma)
$$

(recall that we are now in the $\mathbb{C M}$-space with complex coordinates $z_{\mu}$ ). If this is true, then, on the complex "trajectory" (21), the twistor field is indefinite, and the world line (22) is in fact a focal curve of the congruence. All such congruences can be generated from the corresponding relations for twistor components

$$
\begin{array}{cl}
\tau^{1}=u+w g & =u^{0}(\sigma)+w^{0}(\sigma) g, \\
\tau^{2}=p+v g & =p^{0}(\sigma)+v^{0}(\sigma) g
\end{array}
$$

where a representation of coordinates similar to (16) is used, the distinction being only in the current independence of all four complex coordinates of virtual charge $u^{0}, v^{0},, p^{0}, w^{0}$.

Eliminating, say, the parameter $\sigma$ from the two equations (23), we return to an algebraic constraint of the form (15) with some generating function $\Pi$ and may thus be sure that the arising SFNC will necessarily possess a focal curve, a "world line" of a virtual charge "moving" in the complex extension $\mathbb{C M}$. Note that for a complex "trajectory" (21) the whole focal curve will, generally, have no points of intersection with the real Minkowski cut $\mathbf{M}$. In fact, on $\mathbf{M}$ one can find only the stringlike caustics of the congruence defined by two real (one complex) constraints (19) imposed on four Minkowski coordinates.

Equivalently, one can eliminate the projective spinor component $g$ from the two equations (23) and get, instead of (18), the following generating equation of the complex null cone:

$$
S(\sigma):=Z^{A^{\prime} A}(\sigma) Z_{A^{\prime} A}(\sigma)=0,
$$

where the relative coordinates

$$
Z_{A^{\prime} A}:=x_{A^{\prime} A}-z_{A^{\prime} A}^{0}(\sigma)
$$

come into play, and the points of interest are taken in a real M subspace $z_{A^{\prime} A} \mapsto x_{A^{\prime} A}$. For any $X=\left\{x_{A^{\prime} A}\right\}$, resolving this equation with respect to $\sigma$, one finds a number of branches of the field of "retarded complex time" $\sigma(X)$, any of which possesses the following properties.

1. As in the real case, the field $\sigma(X)$ satisfies the complex eikonal equation (this is easily proved by differentiation of Eq. (24) with respect to coordinates $\left\{x_{A^{\prime} A}\right\}$. 
2. It gives rise to a point "source" $z_{\mu}^{0}(\sigma)$ in the complex space that "influences" at the point $X$.

3 . If one knows $\sigma(X)$, twistor field of the congruence is immediately determined from the generating equations (23) and is constant in value along the elements of the complex null cone (24) connecting the "source" with the corresponding observation point $X$.

In the form (24), the caustics of the corresponding SFNC are evidently determined, instead of (19), by the requirement on the retarded distance to be null,

$$
S^{\prime}(\sigma):=\frac{d S}{d \sigma}=0
$$

The caustic locus equation $S(X)=0$ follows then through elimination of $\sigma$ from (26) and substitution of the result into (24). It is easy to demonstrate that the obtained function $S(X)$ again satisfies the complex eikonal equation .

To do that, let us differentiate, with respect to coordinates $X$, the complex null cone equation (24). Then one gets

$$
\frac{1}{2} \nabla_{C^{\prime} D} S=Z_{C^{\prime} D}-S^{\prime} \nabla_{C^{\prime} D} \sigma .
$$

By virtue of the caustic condition (26), the last term cancels, and one obtains

$$
\frac{1}{4} \nabla_{C^{\prime} D} S \nabla^{C^{\prime} D} S=Z_{C^{\prime} D} Z^{C^{\prime} D}=0,
$$

where one again makes use of the null cone equation (24).

Note that, remarkably, with any SFNC one can associate two functionally independent solutions of the complex eikonal equation. In the null cone representation these are the "retarded" complex time $\sigma(X)$ and the caustic generating function $S(X)$. For the real case these properties are well known: both the "field of retarded time" of an arbitrary moving charge and the function of a "field discontinuity" surface do satisfy the (real) eikonal equation (see, e.g., [16]). However, the principal spinor $g$ is a complex solution to the eikonal equation, even for the case of a charge moving in real space.

\section{The Kerr congruence and its generalization}

Recall that the well-known Kerr SFNC has been first obtained in the work [1. One may consider it as being generated by a virtual pointlike source at rest in complex extension $\mathbb{C M}$, at a separation $a$ from the real slice $\mathbf{M}$. For this case, the null cone equation (24) takes the form

$$
S=(t-\sigma)^{2}-(z+\imath a)^{2}-\rho^{2}=0, \rho:=\sqrt{x^{2}+y^{2}},
$$

the caustics correspond to $\tau=\sigma$ (this is just the solution of the equation $S^{\prime}=0$ ) and, after substitution into (24) - to the condition of caustic locus

$$
-S=(z+\imath a)^{2}+\rho^{2}=0,
$$

which immediately results in the Kerr singular ring

$$
z=0, \quad \rho=a .
$$

The corresponding congruence is twofold, with rectilinear rays as elements of a family of hyperboloids, and the principal spinor $g$ is then determined from Eqs. (23) as follows:

$$
g=\frac{x+\imath y}{\widehat{z} \pm \widehat{r}}, \quad \widehat{r}=\sqrt{(z+\imath a)^{2}+\rho^{2}} .
$$

All this is well known and is presented here only for completeness of the exposition. For details related, in particular, to a possibility of generalizing the Kerr congruence in the way considered below we refer the reader to the old paper by Newman [17.

Let us consider now the case of a virtual charge "moving" uniformly in the complex space with "imaginary" velocity $v v$, that is, let

$$
z_{0}=\sigma, \quad z_{3}=-\imath a+\imath v \sigma, \quad z_{1}=z_{2}=0,
$$

Then the generating null cone equation (24) aquires the form

$$
S=(t-\sigma)^{2}-(z+\imath a-\imath v \sigma)^{2}-\rho^{2}=0,
$$

and the caustic condition reads

$$
\frac{1}{2} S^{\prime}=-(t-\sigma)+\imath v(z+\imath a-\imath v \sigma)=0
$$

Resolving together Eqs.(34) and (35), we find the shape and the dynamics of the caustic ring in the following form [18:

$$
z=0, \quad \rho=\left|-\frac{a}{\sqrt{1+v^{2}}}+\frac{v}{\sqrt{1+v^{2}}} t\right| .
$$

We thus see that the ring collapses into a point at the instant $t_{0}=a / v$ and then starts to expand, uniformly increasing its radius with the velocity

$$
V=\frac{v}{\sqrt{1+v^{2}}},
$$

which is less that the fundamental one $V<c=1$, for any $v$, even for $v>1$. This property is in correspondence with the old result of Bateman [19] who had demonstrated that all of the (generally, stringlike) singularities of an arbitrary solution to the complex eikonal equation lie on a surface propagating with a velocity which is always smaller than the fundamental one.

It is worth noting that the Kerr parameter $a$ loses its important role in the framework under consideration, marking only the instant at which the ring is contracted to a point. Remarkably, this corresponds to the instant when the "virtual" charge pierces through the real physical slice $\mathbf{M}$ and continues afterwards its "motion" in the complex space extension $\mathbb{C M}$.

The deformed SFNC itself is defined via its principal spinor $g$ from the twistor generating equations (23) and turns out to be of the form

$$
g=(1+\imath v) \frac{x+\imath y}{\left(z-z_{t}\right) \pm \widehat{r}}, \quad z_{t}:=-\imath a+\imath v t,
$$


where the time-dependent complex "distance" from the virtual charge is

$$
\widehat{r}=\sqrt{\left(z-z_{t}\right)^{2}+\rho^{2}\left(1+v^{2}\right)} .
$$

Notice that the branching points of the principal spinor $\widehat{r}=0$ just correspond to the caustic ring (36). One can also make sure that the function (38) identically satisfies both the eikonal and the d'Alembert equations.

Let us now calculate the strengths of electromagnetic field associated with the generalized Kerr congruence. To do that, one makes use of the representation (12) and of the particular form of the principal spinor (38). For the field strength components (in cylindrical coordinates) one the gets

$$
\mathcal{E}_{\rho}= \pm \tilde{e} \frac{\rho}{\widehat{r}^{3}}, \quad \mathcal{E}_{z}= \pm \tilde{e} \frac{\left(z-z_{t}\right)}{\widehat{r}^{3}}, \quad \mathcal{E}_{\varphi}=-v \mathcal{E}_{\rho},
$$

where $\tilde{e}:=e\left(1+v^{2}\right)$ while $\Re \overrightarrow{\mathcal{E}}, \Im \overrightarrow{\mathcal{E}}$ correspond to the electric and magnetic field strengths, respectively.

Formally, the obtained field exactly corresponds to that of a point charge moving uniformly in real space along $O Z$ with imaginary velocity. Since Maxwell's linear equations are insensitive to such a formal substitution of a complex entity, one is in fact able to obtain the above field from the very beginning, through the corresponding boost with an imaginary parameter, applied to the ordinary Coulomb field. In his old paper 17, Newman has mentioned a possibility of obtaining such a solution by a complex boost. However, after separation of real (electric) and imaginary (magnetic) parts, the solution (40) looks indeed rather remarkable and possesses the following properties.

1. The fields are two-valued, and the branches transform one into another under a continious path around the singular ring (just as it takes place in the case of the stationary Kerr field). Near the ring, they turn to infinity as $\sim 1 / \delta^{3 / 2}, \delta \rightarrow 0$ being the distance from the ring.

2. The value of the electric charge $e$ (defined from (40) as the flow of he electric field through a surrounding 2-sphere) does not depend on time, on velocity; it is "self-quantized" (in correspondence with the general quantization theorem [15]) and equal to the "elementary" charge of the static (Coulomb) field. The latter occures as the field of the fundamental static solution with $a=0, v=0$, for which the principal spinor $g$ represents the stereographic projection $S^{2} \mapsto \mathbb{C}$ (see, e.g., [5, 6, 9]). (In the adopted dimensionless units, one has $e= \pm 1 / 4)$.

3 . At the very instant when the singularity is pointlike, the field differs from the Coulomb one and possesses, in particular, a vortex electric component $E_{\varphi}$ which at large distances behaves as the radial Coulomblike component, namely, as $\sim 1 / r^{2}$; the magnetic field instantaneously vanishes.

4. The most interesting case is, perhaps, that of the ultrarelativistic contraction/expansion of the singular ring, with the "physical" velocity $V \approx 1$ (this corresponds to the "imaginary" velocity of the virtual charge motion $v>>1$ ). Then at large distances $r>r_{0}, \quad r_{0}=V\left(t-t_{0}\right)$ being the current radius of the singular ring, both the vortex and radial components of the electric and magnetic fields are compressed along OZ and concentrated in a sharp range of angles, $\sin \theta \approx \sqrt{1-V^{2}}$. The same applies to the Pointing vector: it is non-null and may be great in absolute value in the above range of angles.

4. Generally, the magnetic moment, contrary to the electric charge, is variable; for a particular case of Kerr's stationary solution $(v=0)$, it is known to be equal to $e a$, and in view of the value of mass and spin (defined by the metric of the Kerr-Newman solution), corresponds to the Dirac "anomalous" value. This fact has been first noticed by Carter [20] and used further by Newman, Lopes, Burinskii et al. to "model the electron" in the framework of general relativity. At least for slow expansion/contraction, this remarkable property could be preserved (both the spin and the magnetic moment being proportional to the current radius of the singular ring); nonetheless, this conjecture should be verified by a corresponding exact solution to the Einstein-Maxwell set of equations, see below.

\section{Discussion}

The existence of a time-dependent deformation of the Kerr congruence obtained in the paper seems to be a fact of fundamental importance. Indeed, an arbitrary small variation of the parameters of the initial static congruence qualitatively changes its character making it unstable with respect to its scale; as a result, the Kerr singular ring infinitely expands. In turn, the instability of the generating congruence lets one expect similar instability of the Kerr-Schild metric as the corresponding solution to the Einstein equations.

In fact, there are obvious arguments in favour of the existence of such a solution: the general procedure of obtaining exact solutions of the (Maxwell-)Einstein (electro)vacuum equations starting from a shear-free congruence is well known (see, e.g. [12]). Moreover, by analogy with the stationary Kerr case, for such a solution one can conjecture that, during contraction of the "naked" singular ring, a "horizon" would appear at some particular value of the ring current radius, so that one would actually have a continious transition of the naked singularitiy into a rotating black hole. On the other hand, during the ring's subsequent expansion, the "black hole" would throw off its horizon and become a "naked" singularity anew. We hope to prove and examine such solutions in a forthcoming publication.

The observed instability also makes rather problematic the whole activity related to the possible "particlelike" interpretation of the Kerr singular ring (see, e.g., 21, 22]). This becomes quite visual in Newman's representation of a virtual charge "moving" in the $\mathbb{C M}$ 
extension of Minkowski space-time. Actually, the singular (caustic) ring is formed together with the congruence of rays as the trace on $\mathbf{M}$ of the complex null cone of the charge. If we define the electromagnetic field via the congruence itself, as in the above presented scheme, the ring would automatically carry a unit electric charge and a magnetic moment with the right Dirac value of the gyromagnetic factor. However, the mass $M$ and the spin $J=M c a$ would be arbitrary and, to fix the ratio $J / M$, the virtual charge should "oscillate" in a Compton-size "complexified" neighborhood of the real cut M. However, neither in twistor theory nor in properties of the Einstein-Maxwell equations can one find any ground for such restrictions.

To overcome the instability related to the generically stringlike structure of singularities of any complexvalued field on $\mathbf{M}$, one can try to modify the very structure of the background physical space-time, say, by introduction of extra spacelike or timelike dimensions. However, in the framework of the above presented approach, it seems more natural to regard the complex space $\mathbb{C M}$ itself in this capacity; then the transition to physical space-time could be realized via the bilinear mapping suggested in our work [23]. The corresponding construction is motivated algebraically and, in phenomenological respect, naturally introduces an additional compact (phaselike) coordinate which allows for a geometrical explanation of the wave properties of particles [24, 25. It is especially interesting that, in the paradigm of primordial complex space-time, the problem of quantization of characteristics of particlelike singularities is put off to a considerable extent. Indeed, on the $\mathbb{C M}$ background, the fundamental equation of the complex light cone (24) has, generically, a great number of solutions $\left\{\sigma_{N}\right\}$ which fix the corresponding number of observable point singularities in distinct positions $\left\{z_{N}^{0}\right\}$. They all are identical in properties, being in fact one and the same particle at different positions on the unique complexified world line; in 24] these have been called (an ensemble of) duplicons.

Finally, one can pass to studying, in the complex space-time background, the properties of a generic SFNC which, instead of a focal curve - a world line of a virtual charge on the $\mathbb{C M}$ space - is generated by a complex string 18 . The arising structure of singularities seems to be rich enough and capable to describe different types of particlelike formations. We hope to classify them in forthcoming publications.

\section{Acknowledgement}

It is a pleasure for me to thank Profs. G. Neugebauer and G. Schäfer for interesting discussions and valuable remarks on the paper.

\section{References}

[1] G.C. Debney, R.P. Kerr and A. Schild, J. Math. Phys. 10, 1842 (1969).

[2] R. Penrose and W. Rindler "Spinors and Space-Time. Vol.II", Cambridge Univ. Press, Cambridge, 1986.

[3] V.V. Kassandrov and J.A. Rizkallah, "Twistor and "Weak" Gauge Structures in the Framework of Quaternionic Analysis", gr-qc/ 0012109.

[4] V.V. Kassandrov, in: "Space-Time Structure. Algebra and Geometry", ed. D.G. Pavlov et al., Lilia-Print, Moscow, 2007, p. 441, arXiv: 0710.2895.

[5] V.V. Kassandrov, "Algebraic Structure of Space-Time and the Algebrodynamics", Peopl. Fried. Univ. Press, 1992 (in Russian).

[6] V.V. Kassandrov, Grav. \& Cosm. 3, 216 (1995), gr-qc/0007026

[7] G.F. Torres del Castillo, Gen. Rel. Grav. 31, 205 (1999).

[8] V.V. Kassandrov and V.N. Trishin, Gen. Rel. Grav. 36, 1603, (2004), gr-qc/0411120.

[9] V.V. Kassandrov and J.A. Rizcallah, in: "Recent Problems in Field Theory", ed. A.V. Aminova, Kasan Univ. Press, Kasan, 1998, p. 176, gr-qc/9809078.

[10] I. Robinson, J. Math. Phys. 2, 290 (1961).

[11] W. Kinnersley, Phys. Rev. 186, 1335 (1969).

[12] M. Gúrses, F. Gúrsey, J. Math. Phys. 16, 2385 (1974).

[13] R.W. Lind and E.T. Newman, J. Math. Phys. 15, 1103 (1974).

[14] E.T. Newman and J. Winicour, J. Math. Phys. 15, 1113 (1974).

[15] V.V. Kassandrov, in: "Has the Last Word Been Said on Classical Electrodynamics?", ed. A. Chubykalo et al., Rinton Press, 2004, p. 42, physics/0308045

[16] V.A. Fock, "Theory of Space, Time and Gravitation", Fizmatgiz, Moscow, 1961 (in Russian).

[17] E.T. Newman, J. Math. Phys., 14, 102 (1973).

[18] V.V. Kassandrov, in: "Proc. Int. Sch. on Geometry and Analysis", Rostov-na-Donu Univ. Press, 2004, p. 65, gr-qc/0602046.

[19] H. Bateman, "The Mathematical Analysis of Electrical and Optical Wave-Motion", Dover Publ. Inc., 1955.

[20] B. Carter, Phys. Rev. 174, 1559 (1968).

[21] A.Ya. Burinskii, J. Phys. A: Math. Theor. 41, 164069 (2008); arXiv: 0710.4249.

[22] E.T. Newman, Phys. Rev. D65, 104005 (2002), gr-qc/0201055

[23] V.V. Kassandrov, Grav. \& Cosm. 11, 354 (2005), gr-qc/0602088

[24] V.V. Kassandrov, in: "Proc. Int. Conf. Phys. Interpret. Rel. Theory (PIRT-2005)", eds. V.O. Gladyshev et al., Bauman Tech. Univ. Press, Moscow, 2005, p. 42, gr-qc/0602064.

[25] V.V. Kassandrov, Phys. Part. Nuclei, 72, 813, 2009; arxiv: 0907.5425 . 